\title{
Dependency of electrical characteristics on nano gap variation in pinch off lateral gate transistors
}

\begin{abstract}
The variation of electrical characteristics with nano size air gap variation between gates and channel of a pinch off lateral gate transistor were investigated using 3D Technology Computer Aided Design. It is found that smaller nanosize gaps which can be formed by approaching the lateral gates to the channel can improve the switching performance of the device significantly. Devices with different air gap demonstrate same on state current and maximum transconductance of $0.05 \varepsilon S$, however the on/off current ratio (ION/IOFF) is varied by three orders of magnitude. The parameters such as electric field and band energy variation are investigated in order to explain the variation of electrical characteristics by air gap variation.
\end{abstract}

Keyword: Air gap; Junctionless transistor (JLT); Lateral gate (LG); TCAD simulation 